\title{
Characterization of innate immunity genes in the parasitic nematode Brugia malayi
}

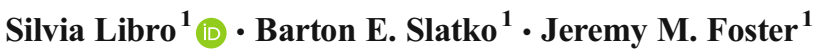

Received: 5 October 2015 / Accepted: 24 December 2015 /Published online: 5 January 2016

(C) The Author(s) 2016. This article is published with open access at Springerlink.com

\begin{abstract}
The filarial nematode Brugia malayi is one of the causative agents of lymphatic filariasis, a neglected tropical disease that affects 120 million people worldwide. The limited effectiveness of available anthelmintics and the absence of a vaccine have prompted extensive research on the interaction between Brugia and its obligate bacterial endosymbiont, Wolbachia. Recent studies suggest that Wolbachia is able to manipulate its nematode host immunity but relatively little is known about the immune system of filarial nematodes. Therefore, elucidation of the mechanisms underlying the immune system of $B$. malayi may be useful for understanding how the symbiotic relationship is maintained and help in the identification of new drug targets. In order to characterize the main genetic pathways involved in B. malayi immunity, we exposed adult female worms to two bacterial lysates (Escherichia coli and Bacillus amyloliquefaciens), dsRNA and dsDNA. We performed transcriptome sequencing of worms exposed to each immune elicitor at two different timepoints. Gene expression analysis of untreated and immune-challenged worms was performed to characterize gene expression patterns associated with each type of immune stimulation. Our results indicate that different immune
\end{abstract}

Presented at the 8 th Congress of the International Symbiosis Society, July 12-18, 2015, Lisbon, Portugal

Electronic supplementary material The online version of this article (doi:10.1007/s13199-015-0374-7) contains supplementary material, which is available to authorized users.

Silvia Libro

libro@neb.com

1 Genome Biology Division, New England Biolabs, Inc., 240 County Road, Ipswich, MA 01938, USA elicitors produced distinct expression patterns in B. malayi, with changes in the expression of orthologs of wellcharacterized $C$. elegans immune pathways such as insulin, TGF- $\beta$, and p38 MAPK pathways, as well as C-type lectins and several stress-response genes.

Keywords Nematode $\cdot$ Brugia $\cdot$ Transcriptomics · Wolbachia $\cdot$ Immunity

\section{Introduction}

The filarial nematode Brugia malayi is one of the causative agents of lymphatic filariasis (elephantiasis), a neglected tropical disease that affects 120 million people in endemic tropical areas. The disease is transmitted to the human host via infected mosquitoes which allow third-stage filarial larvae (L3) to enter the host's bloodstream. Larvae reach maturity in the lymphatic system and reproduce, generating millions of microfilariae that migrate to the capillaries from where they can be ingested by new mosquitos. In the mosquito, microfilariae develop into first-stage larvae, second-stage larvae and then infective thirdstage larvae, and the cycle repeats.

Current treatments such as albendazole and ivermectin rely on mass drug administration (MDA) programs - but they predominantly target larval stages, necessitating a treatment course of up to 10-15 years (Molyneux et al. 2003). Due to this and other limitations of MDA, extensive effort has been devoted to research for alternative treatments. In particular, several projects are currently focusing on understanding the biology of the relationship between B. malayi and its obligate endosymbiont, the alpha-proteobacterium Wolbachia. Wolbachia is present in intracytoplasmic vacuoles in several filarial nematodes and is required for worm fertility and survival (Foster et al. 2013; Taylor et al. 2005) making it a 
promising therapeutic target for filariasis control. One example of anti-filarial strategies exploiting this obligate mutualism is the use of antibiotics against Wolbachia, such as doxycycline, to cause premature death and permanent sterilization of adult worms.

While the mechanisms of the symbiotic relationship are not fully understood, recent studies suggest that apoptosis and autophagy- two conserved cellular pathways essential for homeostasis and innate immunity- are involved in the regulation of Wolbachia titer in filarial nematodes (Landmann et al. 2011; Voronin et al. 2012), suggesting that Wolbachia is able to modulate and evade the host immune system in order to survive. In particular, these studies report extensive apoptosis in germline and somatic cells of embryos, microfilariae, and fourth-stage larvae following antibiotic-mediated depletion of Wolbachia (Landmann et al. 2011) and show that Wolbachia can be recognized and eliminated by autophagy in filarial nematodes and insects (Voronin et al. 2012). Similar to other mutualistic bacterial symbioses, these data are indicative of a cross-talk between Wolbachia and its nematode host immune system. Therefore, determining the mechanisms underlying $B$. malayi immunity can provide critical information to understand how the symbiosis with Wolbachia is maintained.

So far, few studies have focused on the B. malayi immune system and our knowledge is mostly based on sequence similarity with the free-living nematode Caenorhabditis elegans (Engelmann and Pujol 2010; Irazoqui et al. 2010). We initiated experiments aimed at the identification of candidate immune-related genes in the filarial nematode $B$. malayi. Live adult females were exposed to four different immune elicitors (dsRNA, dsDNA, Gram-positive and Gramnegative bacterial lysates) for 24 and $36 \mathrm{~h}$. The expression profiles of treated and un-treated worms were compared and the identity of differentially expressed (DE) transcripts was determined based on current genome annotations. Our results indicate that different immune elicitors produced distinct expression patterns in B. malayi, with changes in the expression of orthologs of well-characterized C. elegans immune pathways such as insulin, transforming growth factor beta (TGF- $\beta$ ), and p38 MAPK pathways, as well as C-type lectins and several stress-response genes.

\section{Materials and methods}

Due to the ex vivo B. malayi experimentation and the consequent challenges associated with the use of live bacteria in culture medium (i.e., risk of death by starvation caused by bacterial overgrowth), bacterial lysates were used to simulate bacterial recognition. Additionally, dsRNA and dsDNA were used in order to stimulate the response to exogenous nucleic acids, based on the evidence that small RNAs are key mediators of antiviral immunity in C. elegans (Wilkins et al. 2005) and that the genome of B. malayi encodes several components of the pathway (Dalzell et al. 2011).

\subsection{Preparation of dsRNA}

The template for the production of dsRNA was LITMUS $28 \mathrm{i}$ (New England Biolabs), a double-stranded cloning/in vitro transcription phagemid vector that has opposing T7 promoters. cDNA was obtained by PCR amplification of a 172 bp region of the vector using Q5 ${ }^{\circledR}$ High-Fidelity $2 \mathrm{X}$ Master Mix (Cat. \#M0492, New England Biolabs) and custom designed primers (IDT): 5-TAA TAC GAC TCA CTA TAG GGC AGA T-3 for the forward primer and 5-TAA TAC GAC TCA CTA TAG GCC TTG ACT AG-3 for the reverse primer. The PCR product was verified by $1 \%$ agarose gel electrophoresis and then purified with the QIAquick PCR Purification Kit (Cat. \#28104, Qiagen) following the manufacturer's protocol but with one extra wash with PE buffer. PCR products were subsequently used to synthesize dsRNA using the HiScribe T7 Quick High Yield RNA Synthesis Kit (Cat. \#E2040S, New England Biolabs) followed by DNase I (Cat. \# AM1907, Ambion) treatment according to the protocol. Concentration and size of dsRNA were assessed respectively with the Qubit ${ }^{\circledR}$ RNA HS Assay Kit (Cat. \#Q32852, Life Technologies) on a Qubit ${ }^{\circledR}$ Fluorometer and with the RNA 6000 Nano Kit (Cat. \#5067-1511, Agilent Technologies) on a Bioanalyzer 2100.

\subsection{Preparation of dsDNA}

Unmethylated dsDNA was obtained from Lambda vector DNA (Cat. \#N3013, New England Biolabs). A 3 ml stock of $2.2 \mathrm{mg} / \mathrm{ml}$ Lambda DNA was diluted five-fold in RNAse free water and sheared to approximately $150 \mathrm{bp}$ using AFA Covaris microtubes (Cat. \#520045, Covaris) on a Covaris S2 sonicator. Size and concentration of dsDNA were assessed by electrophoresis on $1 \%$ agarose gels and confirmed with the Qubit $^{\circledR}$ dsDNA HS (High Sensitivity) Assay Kit (Cat. \#Q32851, Life technologies) and the Agilent DNA $1000 \mathrm{Kit}$ (Cat. \#5067-1504, Agilent Technologies).

\subsection{Bacterial lysates}

Lysates of Escherichia coli and Bacillus amyloliquefaciens were prepared by centrifuging bacterial cultures in a Sharples continuous-flow centrifuge at 17,000 rpm. Cells were then frozen to $-80{ }^{\circ} \mathrm{C}$, thawed overnight at $4{ }^{\circ} \mathrm{C}$ and diluted with water $(1: 3)$ to a final concentration of $33 \mathrm{~g}$ per liter prior to homogenization in a Microfluidizer ${ }^{\mathbb{Q}}$ (Microfluidics) at 20,000 psi. Lysates were stored at $-20{ }^{\circ} \mathrm{C}$, and before being used were thawed at $4{ }^{\circ} \mathrm{C}$ and warmed to $37{ }^{\circ} \mathrm{C}$. 


\subsection{Medium preparation}

A stock of $5 \mathrm{X}$ culture medium was prepared by adding $86 \mathrm{~mL}$ of sterile water, $9.6 \mathrm{~mL}$ of $100 \mathrm{X}$ antibiotic-antimycotic solution containing 10,000 units penicillin, $10 \mathrm{mg}$ streptomycin and $25 \mu \mathrm{g}$ Amphotericin B per ml (Cat. \#A-5955, SigmaAldrich, St Louis, MO, USA) plus $145 \mathrm{mg}$ of L-Glutamine, $1.92 \mathrm{~g}$ of $\mathrm{NaHCO} 3$ and $0.00096 \mathrm{~g}$ of folic acid to $96 \mathrm{~mL}$ of 10X of RPMI-1640 cell culture medium (Cat. \#R1145, Sigma-Aldrich, St Louis, MO, USA). A second stock without the antibiotic was made by adding $89 \mathrm{~mL}$ of sterile water, $1 \mathrm{~mL}$ of 100X glutamine (Cat. \#25030-081, Thermo-Fisher Scientific), $0.38 \mathrm{~g}$ of $\mathrm{NaHCO} 3$ and $0.0002 \mathrm{~g}$ of folic acid to $100 \mathrm{~mL}$ of $10 \mathrm{X}$ of RPMI-1640 cell culture medium. Both stocks were filtered in a Nalgene filtration bottle (Nalgene, Rochester, NY, USA) in a laminar flow hood, stored at $4{ }^{\circ} \mathrm{C}$ and pre-warmed to $37^{\circ} \mathrm{C}$ before use.

\subsection{Experiment set up}

All equipment was sprayed with $70 \%$ ethanol before use under a laminar flow hood. Two sterile 12 -well cell culture plates were set up as follows: each well of the first plate received $200 \mu \mathrm{l}$ of $5 \mathrm{X}$ medium with antibiotic-antimycotic solution. Wells 1 to 4 received $200 \mu \mathrm{l}$ of dsRNA and $600 \mu \mathrm{l}$ of sterile water ( $2.5 \mathrm{uM}$ dsRNA final concentration); wells 5 to 8 received $800 \mu \mathrm{l}$ of sheared DNA ( $2.5 \mathrm{uM}$ dsDNA final concentration) and wells 9 to 12 received $800 \mu$ of sterile water (control plus antibiotic). In the second plate, wells 1 to 8 received $200 \mu \mathrm{l}$ of $5 \mathrm{X}$ medium with antibiotic-antimycotic solution, $300 \mu \mathrm{l}$ of sterile water and $200 \mu \mathrm{l}$ of $B$. amyloliquefaciens (B.a.; wells 1 to 4 ) or E. coli lysate (E.c.; wells 5 to 8 ). Wells 9 to 12 received $200 \mu \mathrm{l}$ of $5 \mathrm{X}$ medium and $800 \mu \mathrm{l}$ of sterile water (control without antibiotic).

Adult B. malayi females were obtained from the NIH/ NIAID Filariasis Research Reagent Resource (FR3) Center. After $2 \mathrm{~h}$ of acclimation in 1X RPMI-1640 cell culture medium with L-glutamine (Invitrogen, Carlsbad, CA, USA) at room temperature, two worms were transferred to each well of the two 12-well cell culture plates using a curved pick. The plates were cultured at $37{ }^{\circ} \mathrm{C}$ in $5 \% \mathrm{CO}_{2}$ under a laminar flow hood. The medium along with each treatment was replaced every $12 \mathrm{~h}$. Worms were inspected approximately every hour during daytime for the duration of the treatment to assess viability and motility, and each worm's behavior was recorded with scores ranging from 3 (normal activity) to 0 (no motility) as described by Keiser (Keiser 2010). Two replicates (i.e., wells) of each treatment were collected at each of the two time points (24 and $36 \mathrm{~h}$ ) and placed in $1.5 \mathrm{~mL}$ DNA LoBind Tubes (Eppendorf, Hamburg, Germany) and stored at -80 . Exceptions were worms exposed to dsDNA, collected after 12 and $16 \mathrm{~h}$, and to E. coli lysate, collected after 20 and
$22 \mathrm{~h}$, due to a rapid decrease in motility compared to the other treatments.

\subsection{RNA extraction and library preparation}

Total RNA was extracted using a modified organic extraction protocol using Trizol (Ambion). Each pair of worms was homogenized in $300 \mu \mathrm{l}$ Trizol using disposable plastic pestles in the original collection tube. A further $200 \mu \mathrm{l}$ of Trizol was used to rinse the pestle in the tube, then $10 \mu \mathrm{l}$ of proteinase $\mathrm{K}$ (Cat. \#P8107, New England Biolabs) was added, followed by $30 \mathrm{~min}$ of incubation at $55^{\circ} \mathrm{C}$. To each tube $270 \mu \mathrm{l}$ of chloroform was added and tubes were incubated for $3 \mathrm{~min}$ at room temperature. Samples were then transferred into Phase Lock Gel Heavy Tubes (Cat. \#0032005152, Eppendorf) with $65 \mu \mathrm{l}$ of fresh Trizol. Samples were centrifuged at $12,000 \mathrm{X} \mathrm{g}$ for 15 min at $4{ }^{\circ} \mathrm{C}$ and the upper aqueous phase was transferred to a DNA LoBind tube. After addition of $5 \mu \mathrm{L}$ of linear acrylamide (Cat. \#E6103, New England Biolabs) and $50 \mu \mathrm{l}$ of sodium acetate $\mathrm{pH}$ 5.2, RNA was recovered by precipitation with isopropanol, washed with $70 \%$ ethanol three times and resuspended in $50 \mu$ of RNAse free water.

Extracted RNA was then treated with DNase I (Cat. \#AM1907, Ambion) and purified using RNA Clean \& Concentrator $^{\mathrm{TM}}-5$ according to the manufacturer's protocol (Cat. \#R1013, Zymo Research). The integrity, purity and concentration of all RNA samples were assessed on an RNA nano chip using an Agilent Bioanalyzer 2100. B. malayi mRNA was isolated using theNEBNext Poly(A) mRNA Magnetic Isolation Module (Cat. \#E7490, New England Biolabs) and transcriptomic libraries were prepared using the NEBNext ${ }^{\circledR}$ Ultra $^{\mathrm{TM}}$ RNA Library Prep Kit for Illumina (Cat. \#E7530, New England Biolabs) and the NEBNext ${ }^{\circledR}$ Multiplex Oligos for Illumina (Index Primers 1-12) (Cat. \# E7335, New England Biolabs) following the kit protocol. Library quality and concentration was assessed using a DNA high sensitivity chip on a Bioanalyzer 2100.

\subsection{Transcriptome sequencing and analysis}

Due to low yield, two libraries were not included in the further analysis: one obtained from worms exposed to medium without antibiotic for $36 \mathrm{~h}$, the other from worms exposed to dsDNA for $16 \mathrm{~h}$. The remaining $22 \mathrm{cDNA}$ libraries (two replicates per treatment), were multiplexed into two pooled samples and sequenced using an Illumina MiSeq platform (Illumina, San Diego, CA, USA) with150 bp paired-end reads. The resulting FASTQ files were imported on a local instance of Galaxy (Blankenberg et al. 2010; Giardine et al. 2005; Goecks et al. 2010) for quality filtering and adaptor trimming. Reads were aligned to the $B$. malayi genome (version: WS247) using TopHat (Galaxy Tool Version 1.5.0) (Trapnell et al. 2009) with default parameters and assembled 
into transcripts with Cufflinks (Galaxy Tool Version 0.0.7) with quartile normalization and multi-read correct options. All the resulting Cufflinks assemblies were then merged with Cuffmerge (Galaxy Tool Version 0.0.6) into a single transcript model. To calculate gene expression levels and statistical significance of expression differences, pairwise comparisons between treatments and controls were performed in Cuffdiff (Galaxy Tool Version 0.0.7) with geometric normalization and false discovery rate (FDR) of 0.01 . The expression level for each transcript was expressed as $\log 2$ fold change of Fragments Per Kilobase of transcript per Million mapped reads (FPKM)-normalized count data.

Transcripts were annotated using a combination of existing annotations retrieved from WormBase and Uniprot databases. Clusters of genes exhibiting significant functional annotation enrichment were identified using the Functional Annotation Clustering tool in DAVID (Database for Annotation, Visualization, and Integrated discovery) (Huang et al. 2008). Among the annotated DE transcripts, putative immune-related genes were identified by manual curation based on literature searches.

\section{Results and discussion}

Transcriptome sequencing of the 22 libraries included in this study yielded a total of $4.2 \times 10^{8}$ read pairs, with an average of $1.9 \times 10^{7}\left( \pm 1.0 \times 10^{7}\right)$ reads per samples. An average of $1.3 \times 10^{7}\left( \pm 8.0 \times 10^{6}\right)$ pairs mapped to the $B$. malayi reference genome, of which $82.7 \%( \pm 12.7 \%)$ were concordant read pairs (See ESM 1).

To account for the effects of the presence of antibiotic/ antimycotic mixture in the medium, gene expression comparisons between controls with and without the antibiotic/ antimycotic mixture were assessed. Transcriptome comparisons of worms exposed to medium with $(\mathrm{C}+\mathrm{A})$ and without antibiotic (C-A) yielded 70 (21 annotated) DE genes after $24 \mathrm{~h}$ incubation and 84 (24 annotated) after $36 \mathrm{~h}$. These transcripts did not show any significant functional enrichment based on the DAVID Functional Annotation Clustering (Fisher exact Pvalue $<0.1$ ) and did not include any known immune mediator (See ESM 2). This was not surprising, as the antibiotics added to the medium are routinely used in the preparation of culture medium to prevent the growth of undesired microorganisms and do not affect Wolbachia (Landmann et al. 2012; Marcellino et al. 2012)

The number of DE transcripts between treatments and controls is shown in Fig. 1. Overall, the two bacterial treatments (22-h E. c. and 24-h B.a.) induced the strongest expression changes relative to $24-\mathrm{h} \mathrm{C}+\mathrm{A}$ with 1008 (390 annotated) and 632 (220 annotated) DE transcripts respectively. This was not entirely unexpected, as the addition of bacterial lysates to the culture resulted in increased turbidity and color change of the

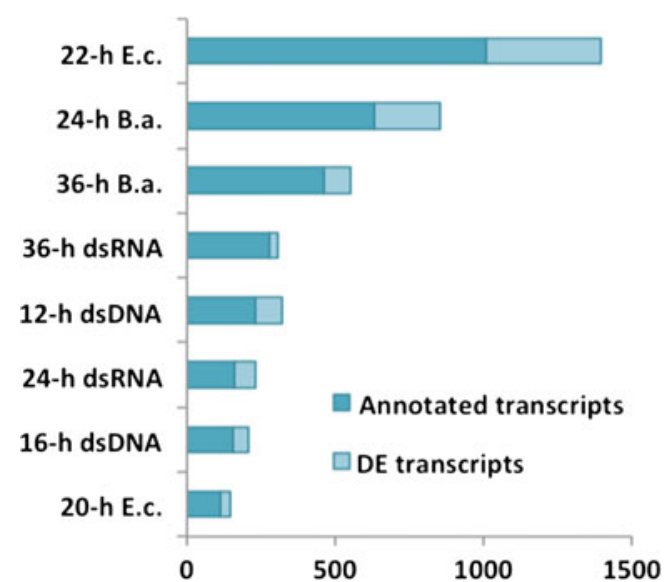

Fig. 1 Differentially expressed (DE) genes between treated worms and controls. Bar charts depicting the number of differentially expressed (adj$\mathrm{p}<0.1)$ transcripts between treated worms and controls. Worms were exposed to the following treatments: dsRNA for 24 and $36 \mathrm{~h}$ (24-h and 36-h dsRNA), dsDNA for 12 and $16 \mathrm{~h}$ (12- and 16-h dsDNA), E. coli lysate for 20 and $22 \mathrm{~h}(20-$ and 22-h E. c.) and B. amyloliquefaciens for 24 and $36 \mathrm{~h}$ (24- and 36-h B. a.). Expression changes were relative to $24-\mathrm{h} \mathrm{C}$ + A (medium plus antibiotic) for all treatments except for 36-h dsRNA and 36-h B. a., which were compared to $36-\mathrm{h} \mathrm{C}+\mathrm{A}$

medium. This likely contributed to the decline in motility in $E$. coli treated worms at 20 and $22 \mathrm{~h}$ post-treatment. Interestingly, worms exposed to $B$. amyloliquefaciens lysate did not exhibit the same alterations in motility. Exposure for $36 \mathrm{~h}$ to $B$. amyloliquefaciens lysate (36-h $B$. a.) and to dsRNA (36-h dsRNA) yielded 462 and 279 DE transcripts (89 and 28 annotated) compared to $36-\mathrm{h} \mathrm{C}+\mathrm{A}$, while comparisons between the dsDNA treatments (12 and 16-h dsDNA) and 24-h C + A yielded 233 and 154 transcripts (89 and 55 annotated).

In general, the top DAVID functional clusters were represented by EGF-like domains, transmembrane proteins, cell adhesion, ion binding, peptidases/metallopeptidase and structural proteins. EGF-like domain was the most overrepresented category in worms exposed to 12-h dsDNA, 24-h dsRNA, 22h E. c. and 24-h dsRNA, while DNA binding, ion binding, structural proteins and transmembrane proteins were enriched in worms exposed to 16-h dsDNA, 36-hs dsRNA, 20-h E. c. and 36-h $B$. $a$. respectively (see EMS2). Two functional clusters were only found in the transcriptome of worms exposed to bacterial treatments, and were represented by immunoglobulin-like (Ig-like), and Leucine rich repeats (LRRs), both candidate immune-related gene families.

\subsection{Candidate immune genes}

In order to identify genes with putative immune functions in B. malayi, the annotations of the DE transcripts were manually curated with particular attention to putative immune-related domains and structures, including C-type lectins, LRRs and Ig-like domain-containing proteins, as well as other known immune regulators in C. elegans. These included components 
of three key immune signaling pathways in C. elegans, p38 MAPK pathway, the insulin pathway and the TGF- $\beta$ pathway (Fig. 2). Based on our data, immune challenge of $B$. malayi triggered alterations in two transcripts with opposite activity on the intestinal p38 MAPK pathway- protein kinase D (Dfk2) and tyrosine-protein phosphatase Vhp-1. Dfk-2 was upregulated in 12-h DNA and 22-h E. c. (0.8 and $1.5 \log _{2}$ fold change, respectively). In C. elegans, Dfk-2 is known to stimulate synthesis of inducible defenses such as C-type lectins and antimicrobials (Hoeckendorf et al. 2012). The expression of the other DE transcript in the pathway, Vhp-1, increased in most treatments (1.5 in 24-h dsRNA, 1.4 in 12-h dsDNA, 0.9 in 20-h E. c., 1.1 in $22-h$ E. c. and 0.8 in $24-h$ B. a.). Vhp-1 is an integrator of the stress response provoked by heavy metals and by infection (Kim et al. 2004; Mizuno et al. 2004) and acts as inhibitor of the p38 MAPK pathway.

A second component of $C$. elegans immunity is the Transforming Growth Factor $\beta$ (TGF- $\beta$ ) pathway, involved in regulation of body morphology and innate immunity in the epidermis (Roberts et al. 2010). The pathway is activated by the binding of Dbl-1, a TGF- $\beta$ homolog produced by neurons, to a transmembrane receptor which - similar to the p38 MAPK pathway - stimulates the expression of antibacterial genes, including lectins, lysozymes and antimicrobials like saposins (Zugasti and Ewbank 2009). Worms exposed to E. coli lysate for $22 \mathrm{~h}$ exhibited down-regulation (-0.9) of Sma-9, a zinc finger transcription factor that acts downstream of Dbl-1 and is thought to confer specific responses to the TGF- $\beta$ pathway activation (Liang et al. 2003). Sma-9 does not seem to be involved in the production of antimicrobials in C. elegans, but rather in the regulation of body size and development (Liang et al. 2003). However, microarray analysis of loss of function mutant worms indicates that C-type lectins and lysozymes are among the targets of Sma-9 (Liang et al. 2007). Interestingly, while the study cited above suggests that Sma9 is a transcriptional activator of C-type lectin genes, our data showed increased expression of C-type lectins in 22-h E.c. treated worms (Fig. 3).

Activation of intestinal insulin signaling pathway by stress or pathogen infection regulates the expression of innate immune genes (Garsin et al. 2003; Singh and Aballay 2009) via nuclear translocation and transcriptional activation of the FOXO transcription factor Daf-16 (Casper et al. 2014). Although no mediator of the insulin pathway was DE in treated worms, the transcription factor Ets-4, that acts as inhibitor of Daf-16, was down-regulated in 22-h E. c. (-1.5). A downstream mediator of the pathway - the DB module protein Dao2 (Yu and Larsen 2001) - was also down-regulated in worms exposed to 22 -h E. $c(-1.9)$ but was up-regulated by $36-\mathrm{h}$ dsRNA (1.3). Dao-2 expression is regulated by Daf-16 and its activity has been associated with oxidative stress response in C. elegans (Shin et al. 2011).

\subsection{Small-RNA pathway}

The small RNA (smRNA) signaling pathway is a conserved mechanism of antiviral defense (siRNA) and posttranscriptional gene regulation (miRNA) via mRNA
Fig. 2 Immune signaling in $C$. elegans host defense. In $C$. elegans, the TGF- $\beta$ pathway regulates body morphology and immunity, the p38 MAPK pathway induces synthesis of C-type lectins and antimicrobials in response to bacterial pathogens and the Daf2-Dafl 6 (aka FOXO) insulin pathway is activated by stress and bacterial infection. Red and blue circles indicate components of the pathways that exhibited respectively up- or downregulation in immune-challenged B. malayi females

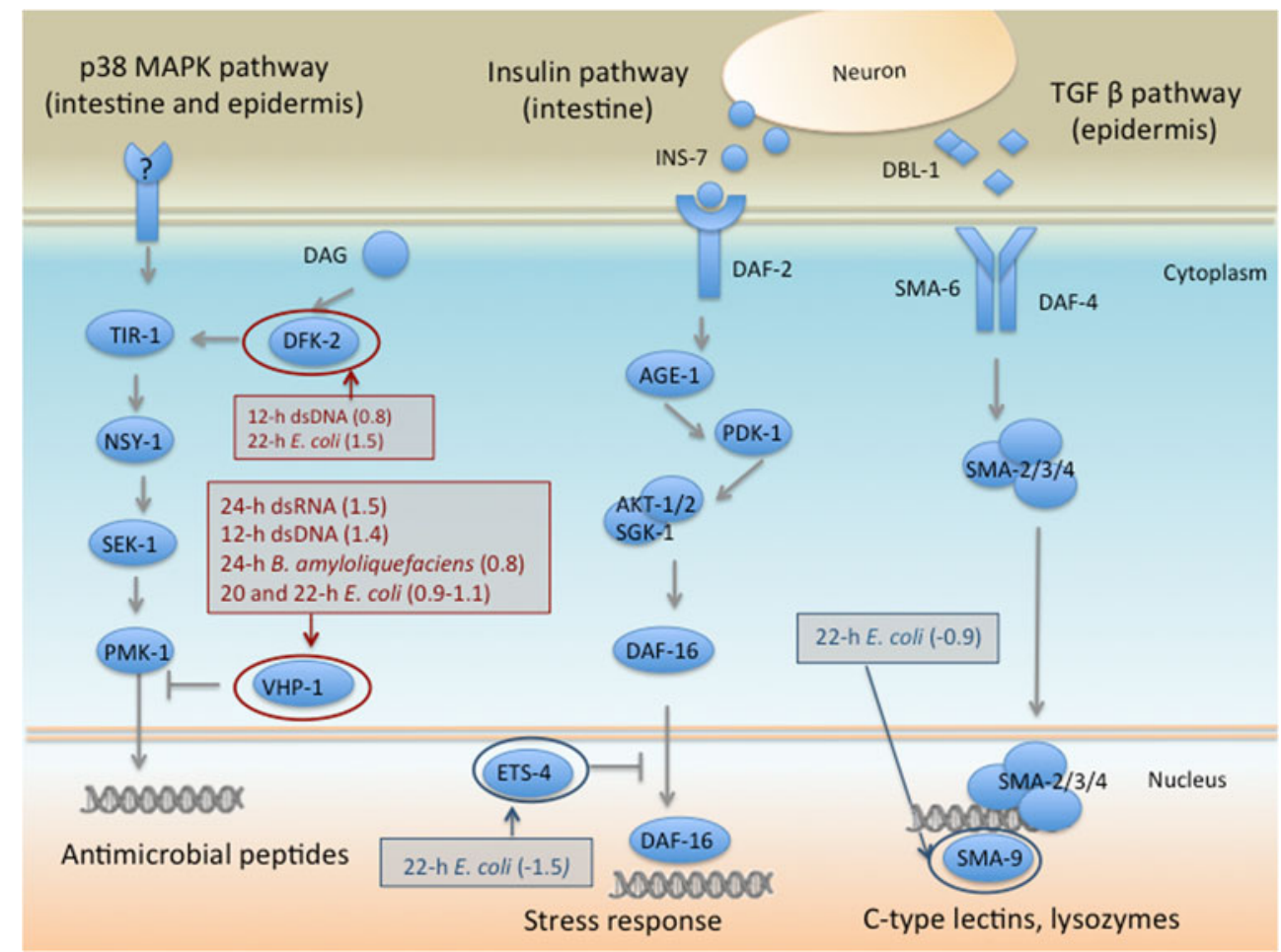


relative
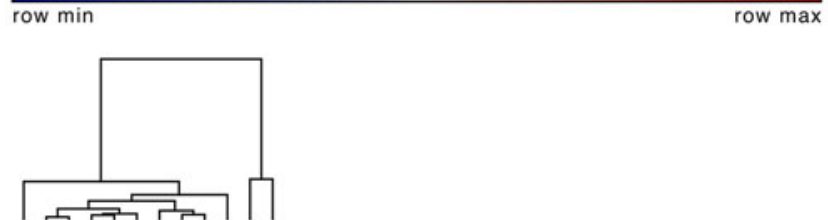

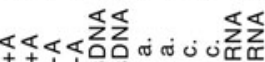

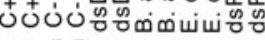

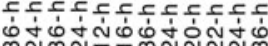

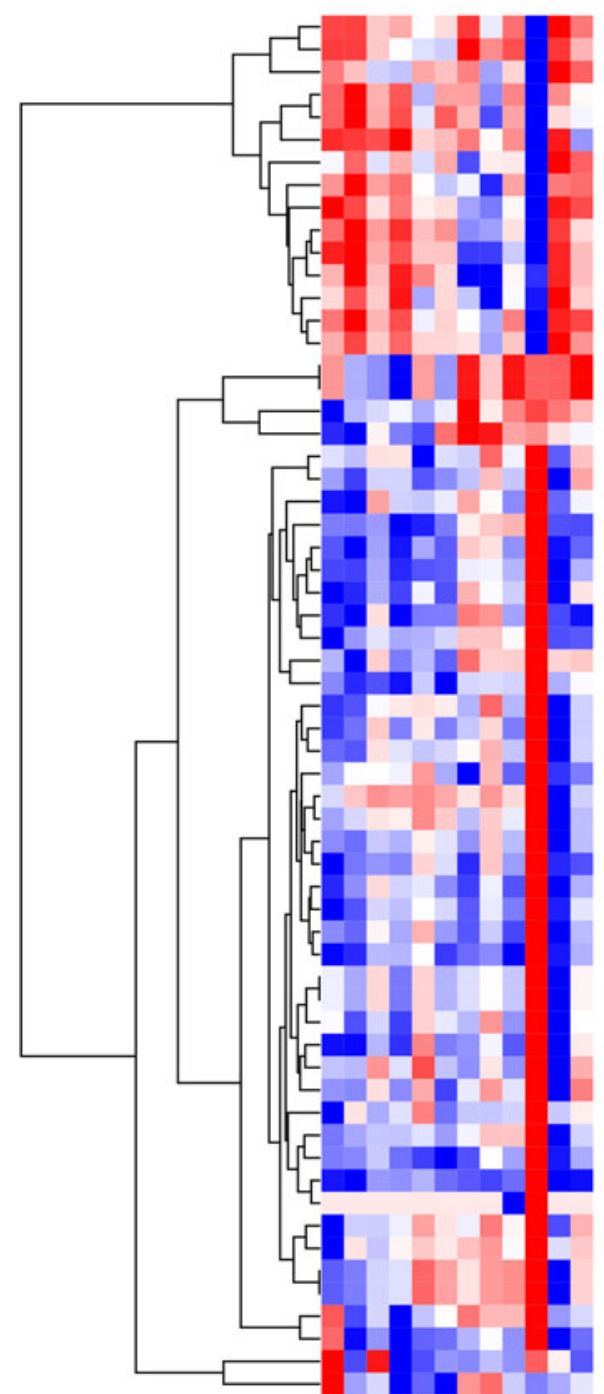

Gene ID

WBGene00223742 WBGene00224039 WBGene00225829 WBGene00223030 WBGene00223031 WBGene00225025 WBGene00221438 WBGene00222060 WBGene00223763 WBGene00226266 WGe 022626 WGen 0227305 BGene0022738 WGene00227253 WBene00226493 WBGene00227849 WBGene00225033 WBGene00225033 WBGene00223471 WBGene00226742 WBGene00220358 WBGene00224494 WBGene00226194 WBGene00225119 WBGene00222209 WBGene00223279 WBGene00227737 WBGene00227118 WBGene0027744 WBGene00227744 WBGene0022542 WBGene0022648 WBGene00221940 WBene00222395 WBaene00227909 WBGene00222860 WBGene00222412 WBGene00225675 WBGene00225404 WBGene0022669 WBGene00223487 WBGene0022713 WBGene00226268 WBGene00227825 WBGene00222335 WBGene00222335 WBGene00227681 WBGene00224538 WBGene0022698 WBGe 022698 WBen 00227595 WBGene00223824 WBGene00225962 WGGene00226345 WBGene00224243 WBGene00227503 WBGene00225328 WBGene0022687 WBGene0022633 WBGene00226337 WBGene00226265 WBGene0022756 WBGene0022685 WBGene00227250

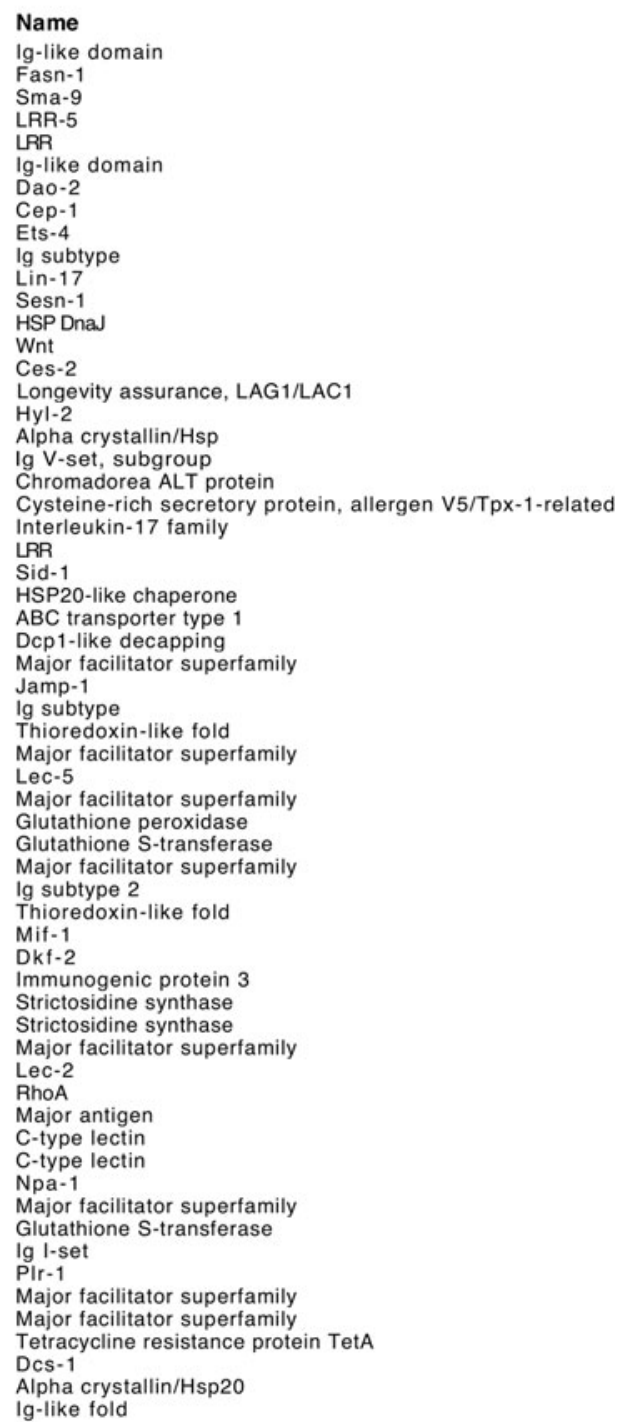

Fig. 3 Clustered heatmap of candidate immune-related genes included in this study. Hierarchical clustering of samples and genes was performed using Pearson correlation metrics. Red color corresponds to up-regulated genes, blue indicates down-regulation. The expression level for each

degradation, translation repression or DNA methylation. smRNAs are generated from either exogenous (during antiviral defense) or genome-encoded (during post-transcriptional regulation) double-stranded RNA that is processed into 21$26 \mathrm{nt}$ fragments by the enzyme Dicer. The mature fragments are then incorporated into the RNA-induced silencing transcript was calculated as $\log 2$ fold change of Fragments Per Kilobase of transcript per Million mapped reads (FPKM)-normalized count data. Only genes with a putative immune function are included in the heatmap

complex (RISC) and then bind to complementary target mRNA sequences promoting their degradation by Argonaute proteins. In C. elegans, the role of miRNAs during stress and pathogen infections has already been reported (Ren and Ambros 2015). While little is known about whether a similar relationship between miRNAs and immunity exists in $B$. 
malayi, Dalzell et al. (Dalzell et al. 2011) highlighted that the genome of $B$. malayi encodes several components of the pathway. Interestingly, only worms exposed to $22-h$ E. c. exhibited signs of increased smRNA signaling, represented by the upregulation of the dsRNA transporter Sid-1 (1.1) and of two transcripts encoding decapping enzymes involved in the turnover of miRNAs (Bosse et al. 2013): Decapping scavenger enzyme 1 (Dcs-1, 1.1) and Dcp1-like decapping enzyme (Dcp-1, 0.7). In C. elegans, Sid-1 enables dsRNA uptake from the culture medium (Shih and Hunter 2011), therefore it is possible that its overexpression indicates internalization of bacterial dsRNA. Argonaute proteins and other components of the small RNA pathways, although present in our dataset, did not show significant expression changes.

\subsection{Wnt pathway}

Wnt signaling is a ubiquitous pathway involved in cell fate determination and development but also in immune modulation in several systems, including C. elegans (Hoeckendorf et al. 2012). In this experiment, Wnt signaling activity was perturbed as indicated by the DE of two activators of the pathway, abnormal cell lineage (Lin-17), a frizzled receptor that initiates the signaling pathway (Irazoqui et al. 2008), and its ligand Wnt. Lin-17 was down-regulated in 22-h E. c. $(-1.4)$, in 24- and in 36-h B. a. $(-1.3$ and -1.2), while Wnt was down-regulated in 22-h E. c. $(-1.5)$ and 24-h B. a. $(-1.0)$. Consistently, worms exposed to 22-h E. c. exhibited upregulation of one inhibitor of the pathway, Cell polarity defective (Plr-1; 0.8). Plr-1, also up-regulated in 36-h dsRNA (0.7) and in 36-h $B$. a. (1.0), is a transmembrane RING finger protein that acts by down-regulating cell surface levels of frizzled receptors including Lin-17 (Moffat et al. 2014). A fourth component of the pathway that acts downstream of frizzled to regulate cell adhesion and cytoskeletal rearrangements, RAS-like GTP-binding protein RhoA, was also upregulated in the 22-h E. c. treatment (1.1). Taken together, these data suggest that exposure to bacterial lysates and in particular 22-h exposure to E. coli, may have an inhibitory effect on the pathway.

\subsection{Apoptosis}

Apoptosis has been extensively studied in C. elegans, where it is required for normal development and homeostasis (reviewed in (Lettre and Hengartner 2006)). In $C$. elegans two Ces (Cell death specification) genes are involved in decision making (Ellis and Horvitz 1991): Ces2 is a basic leucine zipper (bZip) transcription factor that regulates the activity of Ces-1, a Snail family zinc finger transcription factor. Ces-1 blocks cell death by inhibiting the activity of Egl-1, that together with Ced-3, Ced-4 and Ced-9 represents the downstream execution genes
(Metzstein and Horvitz 1999). Apoptosis is initiated by the transcriptional induction of Egl-1 that binds to Ced-9 to release it from Ced-4 (Conradt and Horvitz, 1998; del Peso et al., 2000). After being released from Ced-9, Ced4 can translocate to the nuclear membrane (Chen et al., 2000) and bind to Ced-3 thereby activating it (Yang et al. 1998). The last part of the apoptotic machinery is represented by Ced-1, Ced-2, Ced-5, Ced-6, Ced-7, and Ced-10, involved in the phagocytic engulfment of cell corpses during programmed cell death $(\mathrm{Wu}$ and Horvitz 1998).

In this experiment, two apoptosis mediators were down-regulated: Ces-2, was down-regulated after exposing B. malayi to $E$. coli for $22 \mathrm{~h}(-1.3)$, while $C$. elegans p53-like-1 (Cep-1) was down-regulated in 22-h E. c. $(-3.1)$, in $24-\mathrm{h}$ B. a. $(-2.9)$ and in 16-h dsDNA (-1.9) treatments. Cep-1 is a homolog of the mammalian p53 tumor suppressor protein (Derry et al. 2001) and regulates UVC-induced apoptosis and autophagy by activating the transcription of Egl-1 (Stergiou et al. 2007; Tasdemir et al. 2008). In the absence of stress, p53 is maintained at low levels via proteosome-mediated degradation, while upon stress, p53 levels stabilize and activate stress response programs including cell death (Baruah et al. 2014). The link between apoptosis and stress resistance in C. elegans has been highlighted even further by mutational studies showing that Ced genes, in addition to killing and removing target cells, can also influence environmental stress resistance (Judy et al. 2013). Taken together, these data indicate that the exposure of $B$. malayi to bacterial lysates resulted in down-regulation of several transcripts with a critical role in stress and antibacterial response. While the reason for this down-regulation is not clear, it is possible that it is the result of complex feedback regulatory mechanisms that act to prevent damage resulting from hyperactivation of the immune system. Similar to other organisms, in C. elegans trade-offs exist between immunity and cell metabolism, and activation of immune responses may result in suppression of normal homeostasis to maximize survival during bacterial infection (Ren et al. 2009).

\subsection{Other immune genes}

Other immune-related categories included lectins, putative antimicrobials, nematode allergens and stress-response genes. Consistent with previous studies describing a role in pathogen recognition for C-type lectins in C. elegans (Miltsch et al. 2014; O'Rourke et al. 2006), expression of lectins was high in bacterial treatments, as indicated by the up-regulation of two C-type lectin domain-containing proteins in 22-h E. c. (1.4 and 1.5) and in 24-h B. a. (0.6). Two concanavalin Alike lectins (lec-2 and lec-5) were up-regulated in 22-h E. c. 
(0.9 and 1.0) and 24-h B. a. (0.6), but down-regulated in 20-h E. c. $(-0.7)$. C-type lectins may also be involved in regulating the interaction between the worm and its symbionts. In the marine nematode Laxus oneistus, the C-type lectin Mermaid is secreted on the surface of the worm where it mediates aggregation and attachment of bacterial ectosymbionts to the host's surface (Bulgheresi et al. 2011).

Among putative antimicrobials, a transcript encoding Strictosidine synthase- a conserved enzyme involved in the biosynthesis of an alkaloid with antimicrobial activity (Sohani et al. 2009) was up-regulated in 22-h E. c. (1.1) and down-regulated in 36-h dsRNA $(-0.7)$ while fatty acid synthase Fasn-1, that in C. elegans modulates antimicrobial peptide expression (Lee et al. 2010), was down-regulated in 22-h E. c. $(-0.7)$.

Interestingly, treated worms exhibited increased expression of transcripts classified as allergens based on their ability to trigger an immune response in the human host (Kennedy 2000; Moreno et al. 2011), as well as stress-response genes and xenobiotic detoxicants. See ESM 3 for additional details.

\subsection{Summary}

Overall, the data presented in this study show that the filarial nematode $B$. malayi is able to respond to experimental immune challenges, as revealed by the changes in the expression of several genes that in the free-living nematode $C$. elegans are involved in immunity. Similar to C. elegans, B. malayi possesses an innate immune system that lacks specialized immune cells such as macrophages and phagocytic cells, as well as canonical cytokine and chemokine signaling pathways (i.e., no NF-kB homolog has been found in C. elegans). The three main mechanisms of response to pathogens in C. elegans include avoidance guided by olfactory neurons that "sense" harmful bacteria and fungi (Pradel et al. 2007), mechanical barriers (i.e., cuticle and pharyngeal grinder), and production of inducible defenses such as antimicrobial peptides and reactive oxygen species. It is not clear yet whether the identification of pathogens is mediated by the activity of pathogen recognition receptors (PRRs) like TLR receptors, NOD receptors, and C-type lectins, that are able to recognize and bind to conserved motifs on the surface of pathogens (Akira et al. 2006). To date, only one TLR-like protein has been identified in C. elegans but it does not seem to be directly involved in pathogen recognition. One hypothesis is that surveillance mechanisms act by detecting disruption of critical core processes, such as translational inhibition, generated by bacterial infection (Dunbar et al. 2012).

Another open question is where the immune response takes place in B. malayi. In C. elegans, immunity signaling relies on epithelial cells in the intestine and in the epidermis. Epidermal immunity is induced by pathogen infection or mechanical damage and stimulates the production of antimicrobials via the p38 MAPK and the TGF- $\beta$ pathway. In the intestine, in addition to the p38 MAPK pathway, the insulin signaling pathway mediates stress response and resistance against ingested pathogens. Due to its parasitic lifestyle, B. malayi is likely to be exposed to fewer pathogens compared to free living nematodes, as reflected by the lower diversity of its immune regulators (Murfin et al. 2012). In addition, the need to defend itself from the attack of the human host's immune system may have shaped Brugia's immune mechanisms differently from those of non-parasitic species. For example, the presence of antioxidant enzymes such as glutathione peroxidase in the cuticle has been seen as a strategy to avoid oxidative damage caused by leukocyte-derived reactive oxygen species (Cookson et al. 1992). Interestingly, a recent tissuespecific proteomic analysis revealed high abundance of immune-related proteins such as xenobiotic detoxicants in the intestine of adult B. malayi (Morris et al. 2015), suggesting that the intestine may be as critical as the cuticle in protecting the worm from pathogens and from the attack of human immune cells.

In summary, out of all the treatments included in this study, a 22-h exposure to $E$. coli lysate triggered the strongest response in $B$. malayi, displaying the highest number of DE transcripts compared to controls but also the strongest immune signature. A similar pattern, although less prominent, was found in worms exposed to B. amyloliquefaciens lysates. Surprisingly, exposure to $2.5 \mu \mathrm{M}$ dsDNA appeared to be highly toxic to live adult female worms, as indicated by the sharp decline in motility after only 12 and $16 \mathrm{~h}$ of treatment. It is not clear why dsDNA had such a dramatic effect, as the DNA was obtained from a pure stock and sheared in RNAse-free water. Further studies testing the effects of different dsDNA concentrations need to be conducted to elucidate the mechanisms of apparent DNA toxicity in B. malayi. Further research is also needed in order to distinguish those expression changes exhibited by immune challenged worms that are a true antibacterial response from those that might represent a stress response against high xenobiotic concentrations in the medium.

Future studies will need to focus on the role of Wolbachia during the worm's immune response. Current evidence suggests that Wolbachia acts as powerful modulator of human immunity, as indicated by its ability to delay apoptosis in human polymorphonuclear cells (PMNs) (Bazzocchi et al. 2007). In mosquitos, artificial infection with Wolbachia confers protection against pathogens (Ye et al. 2013) and it is possible that Wolbachia confers increased resistance to stress and pathogens to filarial nematodes as well. However, the same study found no evidence of similar protection in naturally-infected Drosphila populations. Similarly, previous studies did not detect any activation or suppression of inducible defenses in insects following Wolbachia infection (Bourtzis et al. 2000), indicating that this phenomenon is highly dependent on the nature of the symbiosis. As Wolbachia 
resides in cytoplasmic host-derived vacuoles, one possibility is that it avoids phagocytic lysis and degradation either by modifying the vacuoles and/or releasing effector molecules to manipulate the phagocytic pathway. Interestingly, a similar mechanism has been described for Coxiella burnetii, an intracellular bacterium that also develops in phagolysosomal-like vacuoles. C. burnetii interacts with both the phagocytic and autophagic pathways to avoid fusion of the phagosomal vacuole with lysosomes, and uses autophagic vesicles to obtain key nutrients required for its development (Romano et al. 2007). In order to determine whether similar mechanisms underlie the relationship between the autophagy pathway and Wolbachia populations in filarial nematodes, future research is needed to reveal currently unknown aspects of the symbiosis, including the regulatory networks that control Brugia's innate immune pathways.

\section{Conclusions}

Transcriptome profiles of $B$. malayi after immune challenge reveal that the filarial nematode, similarly to $C$. elegans, possesses an array of inducible defenses that are activated upon stress and/or pathogen exposure. Examples of these defenses are conserved signaling pathways including those of $\mathrm{p} 38$ MAPK, TGF- $\beta$ and insulin and small RNAs. It is likely that these pathways act in concert to modulate the tradeoffs between immune activation and cell homeostasis. Even with its challenging technical limitations, this study provides a starting point to address a largely overlooked field, the immunity of filarial nematodes, and offers the potential to improve our understanding of the symbiosis between B. malayi and Wolbachia

\begin{abstract}
Acknowledgments We thank Ashley Luck, William Jack, Clotilde Carlow, Lise Raleigh and Larry McReynolds for critical reading and the helpful comments; Brad Langhorts and Laurence Ettwiller for the bioinformatic support; Laurie Mazzola, Danielle Rivizzigno and Joanna Bybee for the sequencing. We also thank Donald Comb and James Ellard for their continued scientific support. Funding for this project was provided by New England Biolabs, Inc.
\end{abstract}

\section{Compliance with ethical standards}

Competing interests The authors declare that they have no competing interests.

Open Access This article is distributed under the terms of the Creative Commons Attribution 4.0 International License (http:// creativecommons.org/licenses/by/4.0/), which permits unrestricted use, distribution, and reproduction in any medium, provided you give appropriate credit to the original author(s) and the source, provide a link to the Creative Commons license, and indicate if changes were made.

\section{References}

Akira S, Uematsu S, Takeuchi O (2006) Pathogen recognition and innate immunity. Cell 124:783-801. doi:10.1016/j.cell.2006.02.015

Baruah A, Chang H, Hall M, Yuan J, Gordon S, Johnson E, Shtessel LL, Yee C, Hekimi S, Derry WB, Lee SS (2014) CEP-1, the Caenorhabditis elegans 533 homolog, mediates opposing longevity outcomes in mitochondrial electron transport chain mutants. PLoS Genet 10:e1004097. doi:10.1371/journal.pgen.1004097

Bazzocchi C, Comazzi S, Santoni R, Bandi C, Genchi C, Mortarino M (2007) Wolbachia surface protein (WSP) inhibits apoptosis in human neutrophils. Parasite Immunol 29:73-79. doi:10.1111/j.13653024.2006.00915.x

Blankenberg D, Kuster GV, Coraor N, Ananda G, Lazarus R, Mangan M, Nekrutenko A, Taylor J (2010) Galaxy: a web-based genome analysis tool for experimentalists. In: Current protocols in molecular biology. Wiley. doi: 10.1002/0471142727.mb1910s89

Bosse GD, Ruegger S, Ow MC, Vasquez-Rifo A, Rondeau EL, Ambros VR, Grosshans H, Simard MJ (2013) The decapping scavenger enzyme DCS-1 controls microRNA levels in Caenorhabditis elegans. Mol Cell 50:281-287. doi:10.1016/j.molcel.2013.02.023

Bourtzis K, Pettigrew MM, O’Neill SL (2000) Wolbachia neither induces nor suppresses transcripts encoding antimicrobial peptides. Insect Mol Biol 9:635-639. doi:10.1046/j.1365-2583.2000.00224.x

Bulgheresi S, Gruber-Vodicka HR, Heindl NR, Dirks U, Kostadinova M, Breiteneder H, Ott JA (2011) Sequence variability of the pattern recognition receptor Mermaid mediates specificity of marine nematode symbioses ISME J 5:986-998. http://www.nature.com/ismej/ journal/v5/n6/suppinfo/ismej2010198s1.html

Casper SK, Schoeller SJ, Zgoba DM, Phillips AJ, Morien TJ, Chaffee GR, Sackett PC, Peterson FC, Crossgrove K, Veldkamp CT (2014) The solution structure of the forkhead box-ODNA binding domain of Brugia malayi DAF-16a. Proteins Struct Funct Bioinf 82:34903496. doi:10.1002/prot.24701

Chen F, Hersh BM, Conradt B, Zhou Z, Riemer D, Gruenbaum Y, Horvitz HR (2000) Translocation of C. elegans CED-4 to Nuclear Membranes During Programmed Cell Death. Science 287:14851489 doi: $10.1126 /$ science.287.5457.1485

Conradt B, Horvitz HR (1998) The C. elegans Protein EGL-1 Is Required for Programmed Cell Death and Interacts with the Bcl-2-like Protein CED-9. Cell 93:519-529. doi:10.1016/S0092-8674(00) 81182-4

Cookson E, Blaxter ML, Selkirk ME (1992) Identification of the major soluble cuticular glycoprotein of lymphatic filarial nematode parasites (gp29) as a secretory homolog of glutathione peroxidase. Proc Natl Acad Sci U S A 89:5837-5841

Dalzell JJ, McVeigh P, Warnock ND, Mitreva M, Bird DM, Abad P, Fleming CC, Day TA, Mousley A, Marks NJ, Maule AG (2011) RNAi effector diversity in nematodes. PLoS Negl Trop Dis 5:e1176. doi:10.1371/journal.pntd.0001176

del Peso L, González VcM, Inohara N, Ellis RE, Núñez G (2000) Disruption of the CED-9.CED-4 Complex by EGL-1 Is a Critical Step for Programmed Cell Death in Caenorhabditis elegans. J Biol Chem 275:27205-27211

Derry WB, Putzke AP, Rothman JH (2001) Caenorhabditis elegans p53: role in apoptosis, meiosis, and stress resistance. Science 294:591595. doi:10.1126/science. 1065486

Dunbar TL, Yan Z, Balla KM, Smelkinson MG, Troemel ER (2012) C. elegans detects pathogen-induced translational inhibition to activate immune signaling. Cell Host Microbe 11:375-386. doi:10.1016/j. chom.2012.02.008

Ellis RE, Horvitz HR (1991) Two C. elegans genes control the programmed deaths of specific cells in the pharynx. Development 112:591-603 
Engelmann I, Pujol N (2010) Innate immunity in C. elegans. 708:105121. doi:10.1007/978-1-4419-8059-5 6

Foster J, Hoerauf A, Slatko B, Taylor M (2013) The Wolbachia bacterial endosymbionts of filarial nematodes parasitic nematodes. Mol Biol Biochem Immunol 308-336

Garsin DA, Villanueva JM, Begun J, Kim DH, Sifri CD, Calderwood SB, Ruvkun G, Ausubel FM (2003) Long-lived C. elegans daf-2 mutants are resistant to bacterial pathogens. Science 300:1921. doi:10. 1126/science. 1080147

Giardine B, Riemer C, Hardison RC, Burhans R, Elnitski L, Shah P, Zhang Y, Blankenberg D, Albert I, Taylor J, Miller W, Kent WJ, Nekrutenko A (2005) Galaxy: a platform for interactive large-scale genome analysis. Genome Res 15:1451-1455. doi:10.1101/gr. 4086505

Goecks J, Nekrutenko A, Taylor J (2010) Galaxy: a comprehensive approach for supporting accessible, reproducible, and transparent computational research in the life sciences. Genome Biol 11:R86. doi:10. 1186/gb-2010-11-8-r86

Hoeckendorf A, Stanisak M, Leippe M (2012) The saposin-like protein SPP-12 is an antimicrobial polypeptide in the pharyngeal neurons of Caenorhabditis elegans and participates in defence against a natural bacterial pathogen. Biochem J 445:205-212. doi:10.1042/ BJ20112102

Huang DW, Sherman BT, Lempicki RA (2008) Systematic and integrative analysis of large gene lists using DAVID bioinformatics resources. Nat Protocols 4:44-57. http://www.nature.com/nprot/ journal/v4/n1/suppinfo/nprot.2008.211_S1.html

Irazoqui JE, Ng A, Xavier RJ, Ausubel FM (2008) Role for $\beta$-catenin and HOX transcription factors in Caenorhabditis elegans and mammalian host epithelial-pathogen interactions. Proc Natl Acad Sci 105: 17469-17474. doi:10.1073/pnas.0809527105

Irazoqui JE, Urbach JM, Ausubel FM (2010) Evolution of host innate defence: insights from Caenorhabditis elegans and primitive invertebrates. Nat Rev Immunol 10:47-58

Judy ME, Nakamura A, Huang A, Grant H, McCurdy H, Weiberth KF, Gao F, Coppola G, Kenyon C, Kao AW (2013) A shift to organismal stress resistance in programmed cell death mutants. PLoS Genet 9: e1003714. doi:10.1371/journal.pgen.1003714

Keiser J (2010) In vitro and in vivo trematode models for chemotherapeutic studies. Parasitology 137:589-603. doi:10.1017/ S0031182009991739

Kennedy M (2000) The nematode polyprotein allergens/antigens. Parasitol Today 16:373-380. doi:10.1016/S0169-4758(00)01743-9

Kim DH, Liberati NT, Mizuno T, Inoue H, Hisamoto N, Matsumoto K, Ausubel FM (2004) Integration of Caenorhabditis elegans MAPK pathways mediating immunity and stress resistance by MEK-1 MAPK kinase and VHP-1 MAPK phosphatase. Proc Natl Acad Sci U S A 101:10990-10994. doi:10.1073/pnas.0403546101

Landmann F, Voronin D, Sullivan W, Taylor MJ (2011) Anti-filarial activity of antibiotic therapy is due to extensive apoptosis after Wolbachia depletion from filarial nematodes. PLoS Pathog 7: e1002351. doi:10.1371/journal.ppat.1002351

Landmann F, Foster J, Slatko B, Sullivan W (2012) Efficient in vitro RNA interference and immunofluorescence-based phenotype analysis in a human parasitic nematode, Brugia malayi. Parasites Vectors $5: 16$

Lee K-Z, Kniazeva M, Han M, Pujol N, Ewbank J (2010) The fatty acid synthase fasn-1 acts upstream of WNK and Ste20/GCK-VI kinases to modulate antimicrobial peptide expression in C. elegans epidermis. Virulence 1:113-122. doi:10.4161/viru.1.3.10974

Lettre G, Hengartner MO (2006) Developmental apoptosis in C. elegans: a complex CEDnario. Nat Rev Mol Cell Biol 7:97-108

Liang J, Lints R, Foehr ML, Tokarz R, Yu L, Emmons SW, Liu J, SavageDunn C (2003) The Caenorhabditis elegans schnurri homolog sma9 mediates stage- and cell type-specific responses to DBL-1 BMP- related signaling. Development 130:6453-6464. doi:10.1242/dev. 00863

Liang J, Yu L, Yin J, Savage-Dunn C (2007) Transcriptional repressor and activator activities of SMA-9 contribute differentially to BMPrelated signaling outputs. Dev Biol 305:714-725. doi:10.1016/j. ydbio.2007.02.038

Marcellino C, Gut J, Lim KC, Singh R, McKerrow J, Sakanari J (2012) WormAssay: a novel computer application for whole-plate motionbased screening of macroscopic parasites. PLoS Negl Trop Dis 6: e1494. doi:10.1371/journal.pntd.0001494

Metzstein MM, Horvitz HR (1999) The C. elegans cell death specification gene ces- 1 encodes a snail family zinc finger protein. Mol Cell 4:309-319. doi:10.1016/S1097-2765(00)80333-0

Miltsch SM, Seeberger PH, Lepenies B (2014) The C-type lectin-like domain containing proteins $\mathrm{Clec}-39$ and $\mathrm{Clec}-49$ are crucial for Caenorhabditis elegans immunity against Serratia marcescens infection. Dev Comp Immunol 45:67-73. doi:10.1016/j.dci.2014.02. 002

Mizuno T, Hisamoto N, Terada T, Kondo T, Adachi M, Nishida E, Kim DH, Ausubel FM, Matsumoto K (2004) The Caenorhabditis elegans MAPK phosphatase VHP-1 mediates a novel JNK-like signaling pathway in stress response. EMBO J 23:2226-2234. doi:10. 1038/sj.emboj.7600226

Moffat LL, Robinson RE, Bakoulis A, Clark SG (2014) The conserved transmembrane RING finger protein PLR-1 downregulates Wnt signaling by reducing frizzled, ror and ryk cell-surface levels in $C$. elegans. Development 141:617-628. doi:10.1242/dev.101600

Molyneux DH, Bradley M, Hoerauf A, Kyelem D, Taylor MJ (2003) Mass drug treatment for lymphatic filariasis and onchocerciasis. Trends Parasitol 19:516-522. doi:10.1016/j.pt.2003.09.004

Moreno Y, Gros P-P, Tam M, Segura M, Valanparambil R, Geary TG, Stevenson MM (2011) Proteomic analysis of excretory-secretory products of Heligmosomoides polygyrus assessed with nextgeneration sequencing transcriptomic information. PLoS Negl Trop Dis 5:e1370. doi:10.1371/journal.pntd.0001370

Morris CP, Bennuru S, Kropp LE, Zweben JA, Meng Z, Taylor RT, Chan K, Veenstra TD, Nutman TB, Mitre E (2015) A proteomic analysis of the body wall, digestive tract, and reproductive tract of Brugia malayi. PLoS Negl Trop Dis 9:e0004054. doi:10.1371/journal.pntd. 0004054

Murfin KE, Dillman AR, Foster JM, Bulgheresi S, Slatko BE, Sternberg PW, Goodrich-Blair H (2012) Nematode-bacterium symbioses-cooperation and conflict revealed in the "Omics" age. Biol Bull 223: $85-102$

O'Rourke D, Baban D, Demidova M, Mott R, Hodgkin J (2006) Genomic clusters, putative pathogen recognition molecules, and antimicrobial genes are induced by infection of $C$. elegans with $M$. nematophilum. Genome Res 16:1005-1016. doi:10.1101/gr. 50823006

Pradel E, Zhang Y, Pujol N, Matsuyama T, Bargmann CI, Ewbank JJ (2007) Detection and avoidance of a natural product from the pathogenic bacterium Serratia marcescens by Caenorhabditis elegans. Proc Natl Acad Sci 104:2295-2300. doi:10.1073/pnas.0610281104

Ren Z, Ambros VR (2015) Caenorhabditis elegans microRNAs of the let-7 family act in innate immune response circuits and confer robust developmental timing against pathogen stress. Proc Natl Acad Sci 112:E2366-E2375. doi:10.1073/pnas.1422858112

Ren M, Feng H, Fu Y, Land M, Rubin CS (2009) Protein kinase D is an essential regulator of $C$. elegans innate immunity. Immunity 30 : 521-532. doi:10.1016/j.immuni.2009.03.007

Roberts AF, Gumienny TL, Gleason RJ, Wang H, Padgett RW (2010) Regulation of genes affecting body size and innate immunity by the DBL-1/BMP-like pathway in Caenorhabditis elegans. BMC Dev Biol 10:61. doi:10.1186/1471-213X-10-61

Romano PS, Gutierrez MG, Beron W, Rabinovitch M, Colombo MI (2007) The autophagic pathway is actively modulated by phase II 
Coxiella burnetii to efficiently replicate in the host cell. Cell Microbiol 9:891-909. doi:10.1111/j.1462-5822.2006.00838.x

Shih JD, Hunter CP (2011) SID-1 is a dsRNA-selective dsRNA-gated channel. RNA 17:1057-1065. doi:10.1261/rna.2596511

Shin H, Lee H, Fejes AP, Baillie DL, Koo HS, Jones SJ (2011) Gene expression profiling of oxidative stress response of $C$. elegans aging defective AMPK mutants using massively parallel transcriptome sequencing. BMC Res Notes 4:34. doi:10.1186/1756-0500-4-34

Singh V, Aballay A (2009) Regulation of DAF-16-mediated Innate Immunity in Caenorhabditis elegans. J Biol Chem 284:35580 35587. doi:10.1074/jbc.M109.060905

Sohani MM, Schenk PM, Schultz CJ, Schmidt O (2009) Phylogenetic and transcriptional analysis of a strictosidine synthase-like gene family in Arabidopsis thaliana reveals involvement in plant defence responses. Plant Biol 11:105-117. doi:10.1111/j.1438-8677.2008.00139.x

Stergiou L, Doukoumetzidis K, Sendoel A, Hengartner MO (2007) The nucleotide excision repair pathway is required for UV-C-induced apoptosis in Caenorhabditis elegans. Cell Death Differ 14:11291138. http://www.nature.com/cdd/journal/v14/n6/suppinfo/ 4402115s1.html

Tasdemir E, Maiuri MC, Galluzzi L, Vitale I, Djavaheri-Mergny M, D'Amelio M, Criollo A, Morselli E, Zhu C, Harper F, Nannmark U, Samara C, Pinton P, Vicencio JM, Carnuccio R, Moll UM, Madeo F, Paterlini-Brechot P, Rizzuto R, Szabadkai G, Pierron G, Blomgren K, Tavernarakis N, Codogno P, Cecconi F, Kroemer G (2008) Regulation of autophagy by cytoplasmic p53. Nat Cell Biol 10:676-687. http://www.nature.com/ncb/journal/v10/n6/suppinfo/ ncb1730_S1.html

Taylor MJ, Bandi C, Hoerauf A (2005) Wolbachia.Bacterial endosymbionts of filarial nematodes. In: J.R. Baker RM, Rollinson D (eds)
Advances in parasitology, vol 60. Academic Press, pp 245-284. doi: 10.1016/S0065-308X(05)60004-8

Trapnell C, Pachter L, Salzberg SL (2009) TopHat: discovering splice junctions with RNA-Seq. Bioinformatics 25:1105-1111. doi:10. 1093/bioinformatics/btp120

Voronin D, Cook DAN, Steven A, Taylor MJ (2012) Autophagy regulates Wolbachia populations across diverse symbiotic associations. Proc Natl Acad Sci 109:E1638-E1646. doi:10.1073/pnas.1203519109

Wilkins C, Dishongh R, Moore SC, Whitt MA, Chow M, Machaca K (2005) RNA interference is an antiviral defence mechanism in Caenorhabditis elegans. Nature 436:1044-1047

Wu Y-C, Horvitz HR (1998) C. elegans phagocytosis and cellmigration protein CED-5 is similar to human DOCK180. Nature 392:501-504

Yang X, Chang HY, Baltimore D (1998) Essential role of CED-4 oligomerization in CED-3 activation and apoptosis. Science 281:13551357

Ye YH, Woolfit M, Rancès E, O'Neill SL, McGraw EA (2013) Wolbachia Associated Bacterial Protection in the Mosquito Aedes aegypti. PLoS Negl Trop Dis 7:e2362. doi:10.1371/journal.pntd.0002362

Yu H, Larsen PL (2001) DAF-16-dependent and independent expression targets of DAF-2 insulin receptor-like pathway in Caenorhabditis elegans include FKBPs1. J Mol Biol 314:1017-1028. doi:10.1006/ jmbi.2000.5210

Zugasti O, Ewbank JJ (2009) Neuroimmune regulation of antimicrobial peptide expression by a noncanonical TGF-[beta] signaling pathway in Caenorhabditis elegans epidermis. Nat Immunol 10:249-256. http:/www.nature.com/ni/journal/v10/ n3/suppinfo/ni.1700 S1.html 